\title{
Expansions of o-minimal structures by sparse sets
}

\author{
by \\ Harvey Friedman and Chris Miller (Columbus, $\mathrm{OH}$ )
}

\begin{abstract}
Given an o-minimal expansion $\mathfrak{R}$ of the ordered additive group of real numbers and $E \subseteq \mathbb{R}$, we consider the extent to which basic metric and topological properties of subsets of $\mathbb{R}$ definable in the expansion $(\mathfrak{R}, E)$ are inherited by the subsets of $\mathbb{R}$ definable in certain expansions of $(\Re, E)$. In particular, suppose that $f\left(E^{m}\right)$ has no interior for each $m \in \mathbb{N}$ and $f: \mathbb{R}^{m} \rightarrow \mathbb{R}$ definable in $\mathfrak{R}$, and that every subset of $\mathbb{R}$ definable in $(\Re, E)$ has interior or is nowhere dense. Then every subset of $\mathbb{R}$ definable in $(\mathfrak{R},(S))$ has interior or is nowhere dense, where $S$ ranges over all nonempty subsets of all cartesian products $E^{k}(k \geq 1)$. The same holds true with "nowhere dense" replaced by any of "null" (in the sense of Lebesgue), "countable", "a finite union of discrete sets", or "discrete". We use this (together with a result of L. van den Dries) to give an example of an expansion of the real field that defines an isomorphic copy of the ordered ring of integers, yet does not define $\mathbb{Z}$.
\end{abstract}

Given a first-order expansion $\mathfrak{R}$ of the real line and $E \subseteq \mathbb{R}$, let $(\Re, E)^{\#}$ denote the structure $(\mathfrak{R},(S))$, where $S$ ranges over all nonempty subsets of all cartesian products $E^{k}$ ( $k$ ranging over all positive integers). Here is the main technical result of this paper:

Theorem A. Let $\mathfrak{R}$ be an o-minimal expansion of $(\mathbb{R},<,+)$. Let $E \subseteq \mathbb{R}$ be such that, for every $m \in \mathbb{N}$ and $f: \mathbb{R}^{m} \rightarrow \mathbb{R}$ definable in $\mathfrak{R}$, the closure of $f\left(E^{m}\right)$ is nowhere dense. Then every subset of $\mathbb{R}$ definable in $(\mathfrak{R}, E)^{\#}$ either has interior or is nowhere dense. The same holds true with "nowhere dense" replaced (uniformly) by any of "null" (in the sense of Lebesgue), "countable", "a finite union of discrete sets", or "discrete".

(Throughout, "definable" means "definable with parameters" in the structure under consideration. An expansion of the real line $(\mathbb{R},<)$ is

2000 Mathematics Subject Classification: Primary 03C64.

The first author was supported by NSF Grant No. DMS-734164. The second author was supported by NSF Grant No. DMS-9896225 and, in part, by the Mathematical Sciences Research Institute (Berkeley). 
o-minimal if every definable subset of $\mathbb{R}$ is a finite union of points and open intervals. A subset of $\mathbb{R}$ is discrete if every point of the set is isolated.) Of course, when considering the "nowhere dense" version of Theorem A, the phrase "the closure of" can be deleted. An extra assumption on $E$ ensures that many basic metric and topological properties of subsets of $\mathbb{R}$ definable in $(\mathfrak{R}, E)^{\#}$ are determined by the properties of the subsets of $\mathbb{R}$ definable in $(\Re, E)$.

Definition. Given an expansion $\mathfrak{R}$ of the real line, a set $E \subseteq \mathbb{R}$ is $\mathfrak{R}$-sparse (or sparse with respect to $\mathfrak{R}$ ) if, for each $n \in \mathbb{N}$ and definable $f: \mathbb{R}^{n} \rightarrow \mathbb{R}$, the image $f\left(E^{n}\right)$ has no interior.

Obviously, if $E$ has cardinality less than that of the continuum, then $E$ is sparse with respect to every expansion of the real line, but there are more exotic examples. (After the proof of Theorem A, there is a brief discussion of sparseness.)

Theorem B. Let $\mathfrak{R}$ be an o-minimal expansion of $(\mathbb{R},<,+)$ and $E \subseteq \mathbb{R}$ be $\mathfrak{R}$-sparse. If every subset of $\mathbb{R}$ definable in $(\mathfrak{R}, E)$ has interior or is nowhere dense, then every subset of $\mathbb{R}$ definable in $(\mathfrak{R}, E)^{\#}$ has interior or is nowhere dense. The same holds true with "nowhere dense" replaced by any of "null", "countable", "a finite union of discrete sets", or "discrete".

Proof. Suppose that every subset of $\mathbb{R}$ definable in $(\mathfrak{R}, E)$ has interior or is nowhere dense. Since $E$ is $\mathfrak{R}$-sparse, $f\left(E^{m}\right)$ is nowhere dense for every $m \in \mathbb{N}$ and function $f: \mathbb{R}^{m} \rightarrow \mathbb{R}$ definable in $\mathfrak{R}$. Now apply Theorem A. We do one other version, and leave the rest to the reader. Suppose that every subset of $\mathbb{R}$ definable in $(\mathfrak{R}, E)$ has interior or is null. Then every subset of $\mathbb{R}$ definable in $(\mathfrak{R}, E)$ has interior or is nowhere dense. (If $A \subseteq \mathbb{R}$ is dense in some open interval $I$, then at most one of $I \cap A, I \backslash A$ is null.) Hence, $\operatorname{cl}\left(f\left(E^{m}\right)\right)$ has no interior-and thus is null-for every $m \in \mathbb{N}$ and function $f: \mathbb{R}^{m} \rightarrow \mathbb{R}$ definable in $\mathfrak{R}$. Now apply Theorem A.

REMARK. For any expansion of $(\mathbb{R},<)$, the condition that every definable subset of $\mathbb{R}$ has interior or is meager (in the sense of Baire) is equivalent to the condition that every definable subset of $\mathbb{R}$ has interior or is nowhere dense. (If $A \subseteq \mathbb{R}$ is dense in some open interval $I$, then at most one of $I \cap A$, $I \backslash A$ is meager.)

EXAMPLE. For any expansion $\mathfrak{R}$ of $(\mathbb{R},<)$, the condition that every definable subset of $\mathbb{R}$ be the union of an open set and a discrete set is equivalent to the condition that $\mathfrak{R}$ be locally o-minimal, that is, given any definable $A \subseteq \mathbb{R}$ and $x \in \mathbb{R}$, there is an open interval $I$ containing $x$ such $I \cap A$ is a finite union of points and open intervals. As an application of Theorem A, we deduce that the structure $(\mathbb{R},<,+, \mathbb{Z})^{\#}$ is locally o-minimal. To see this, let $f: \mathbb{R}^{n} \rightarrow \mathbb{R}$ be definable in $(\mathbb{R},<,+)$. Then $f$ is semilinear, and there is a fi- 
nite partition $C_{1}, \ldots, C_{l}$ of $\mathbb{R}^{n}$ and maps $T_{1}, \ldots, T_{l} \in \mathbb{R}+\sum_{i=1}^{n} \mathbb{Q} x_{i}$ such that $f\left|C_{i}=T_{i}\right| C_{i}$ for $i=1, \ldots, l$. The image of $\mathbb{Z}^{n}$ under any $T \in \mathbb{R}+\sum_{i=1}^{n} \mathbb{Q} x_{i}$ is closed and discrete. A finite union of closed discrete sets is closed and discrete, so $f\left(\mathbb{Z}^{n}\right)$ is closed and discrete. Apply Theorem A.

Example. Put $2^{\mathbb{Z}}=\left\{2^{k}: k \in \mathbb{Z}\right\}$. In [1], van den Dries shows that every subset of $\mathbb{R}$ definable in $\left(\mathbb{R},+, \cdot, 2^{\mathbb{Z}}\right)$ is the union of an open set and finitely many discrete sets, and that $\operatorname{Th}\left(\mathbb{R},+, \cdot, 2^{\mathbb{Z}}\right)$ is decidable. By Theorem B, every subset of $\mathbb{R}$ definable in $\left(\mathbb{R},+, \cdot, 2^{\mathbb{Z}}\right) \#$ is the union of an open set and finitely many discrete sets; hence, the same is true of the structure $(\mathbb{R},+, \cdot, X)$, with $X=\left\{\left(2^{j}, 2^{k}, 2^{j k}\right): j, k \in \mathbb{Z}\right\}$. Now, $X$ is the graph of the restriction to $2^{\mathbb{Z}} \times 2^{\mathbb{Z}}$ of the function

$$
(x, y) \mapsto 2^{\left(\log _{2} x\right)\left(\log _{2} y\right)}:(0, \infty) \times(0, \infty) \rightarrow \mathbb{R}
$$

so $(\mathbb{R},+, \cdot, X)$ defines an isomorphic copy of $(\mathbb{Z},<,+, \cdot, 0,1)$. Hence, although the topology of reducts of $\left(\mathbb{R},+, \cdot, 2^{\mathbb{Z}}\right) \#$ is uniformly controlled, so to speak, by that of $\left(\mathbb{R},+, \cdot, 2^{\mathbb{Z}}\right)$, the model theory of reducts of $\left(\mathbb{R},+, \cdot, 2^{\mathbb{Z}}\right) \#$ can be much wilder than that of $\left(\mathbb{R},+, \cdot, 2^{\mathbb{Z}}\right)$. Note also that $\mathbb{Q}$ is not definable in $\left(\mathbb{R},+, \cdot, 2^{\mathbb{Z}}\right)^{\#}$, hence neither is $\mathbb{Z}$.

EXAmple. Let Fib denote the set of all Fibonacci numbers $\left\{a_{n}: n \geq 1\right\}$. Recall that for every $n \geq 1$, we have

$$
a_{n}= \begin{cases}\left(\varphi^{n}-\varphi^{-n}\right) / \sqrt{5} & \text { if } n \text { is even } \\ \left(\varphi^{n}+\varphi^{-n}\right) / \sqrt{5} & \text { if } n \text { is odd }\end{cases}
$$

where $\varphi=(1+\sqrt{5}) / 2$. Hence, Fib is definable in $\left(\mathbb{R},+, \cdot, \varphi^{\mathbb{Z}}\right)$. (We thank R. Dougherty for pointing this out.) Again by [1], every subset of $\mathbb{R}$ definable in $\left(\mathbb{R},+, \cdot, \varphi^{\mathbb{Z}}\right)$ is the union of an open set and finitely many discrete sets, so the same is true of $(\mathbb{R},+, \cdot$, Fib). By Theorem $B$, every subset of $\mathbb{R}$ definable in $(\mathbb{R},+, \cdot, \mathrm{Fib})^{\#}$, as well as every subset of $\mathbb{R}$ definable in $\left(\mathbb{R},+, \cdot, \varphi^{\mathbb{Z}}\right)^{\#}$, is the union of an open set and finitely many discrete sets.

Acknowledgements. The results in this paper grew out of Miller's participation in the Model Theory of Fields program at the Mathematical Sciences Research Institute (Berkeley) during the Spring of 1998. He thanks the Institute for its support and hospitality. In an e-mail communication (August, 1998) to Miller, Friedman introduced a method of infinitary quantifier elimination that established the "nowhere dense" and "countable" versions of the statement of Theorem A, and the corresponding "card $(E)<\operatorname{card}(\mathbb{R})$ " versions of Theorem B. The proof presented in this paper is an adaptation of the method.

Throughout the rest of this paper, $\mathfrak{R}$ denotes a fixed, but arbitrary, o-minimal expansion of $(\mathbb{R},<,+)$ and $E$ denotes a fixed subset of $\mathbb{R}$. To avoid trivialites, we assume that $E$ is infinite. 
Proof of Theorem A. We shall establish an a priori stronger result, namely, that the statement of Theorem A holds for a certain expansion (in the sense of definability) of $(\Re, E)^{\#}$. We outline the structure of the proof now. For each $n \in \mathbb{N}$, we introduce collections $\mathcal{S}_{n}$ and $\mathcal{T}_{n}$ of subsets of $\mathbb{R}^{n}$. (We regard $\mathbb{R}^{0}$ as the one-point space $\{0\}$, and a function $f: \mathbb{R}^{0} \rightarrow \mathbb{R}$ as the corresponding constant $f(0)$.) We postpone their definitions, but list here their relevant properties, and then immediately deduce Theorem A.

(A) $\mathcal{T}_{n}$ is a boolean algebra, containing every subset of $\mathbb{R}^{n}$ definable in $\mathfrak{R}$, as well as every set of the form $F^{-1}(P)$, where $m \in \mathbb{N}, P \subseteq E^{m}$, and $F: \mathbb{R}^{n} \rightarrow \mathbb{R}^{m}$ is definable in $\mathfrak{R}$. (B) Every element of $\mathcal{T}_{n}$ is a finite union of elements of $\mathcal{S}_{n}$. (C) For every $A \in \mathcal{S}_{n+1}$, the projection of $A$ on the first $n$ coordinates belongs to $\mathcal{T}_{n}$. (D) For every $A \in \mathcal{S}_{1}$, if $A$ has no interior, then there exist $m \in \mathbb{N}$ and a function $f: \mathbb{R}^{m} \rightarrow \mathbb{R}$ definable in $\mathfrak{R}$ such that $A \subseteq \operatorname{cl}\left(f\left(E^{m}\right)\right)$.

Remark. Property (D) is trivial if $\mathfrak{R}$ defines a bijection between a bounded and an unbounded interval - in particular, if $\Re$ expands the fieldand some function $f: \mathbb{R}^{m} \rightarrow \mathbb{R}$ such that $f\left(E^{m}\right)$ is somewhere dense.

Proof of Theorem A. Consider the structure $(\mathbb{R},(Y))$ where $Y$ ranges over all elements of all $\mathcal{S}_{k}, k \geq 1$. By Properties (A), (B) and (C), for every $n \in \mathbb{N}$, every subset of $\mathbb{R}^{n}$ definable in $(\mathfrak{R}, E)^{\#}$ is definable in $(\mathbb{R},(Y))$, and every subset of $\mathbb{R}^{n}$ definable in $(\mathbb{R},(Y))$ is a finite union of elements of $\mathcal{S}_{n}$. Hence, every subset of $\mathbb{R}$ definable in $(\mathfrak{R}, E) \#$ is a finite union of elements of $\mathcal{S}_{1}$. Now apply (D). (Note that finite unions of closed discrete sets are closed and discrete.)

We now work toward the establishment of Properties (A) through (D). The variables $j, k, l, m, n$ range over $\mathbb{N}$. We identify $\mathbb{R}^{m} \times \mathbb{R}^{n}$ with $\mathbb{R}^{m+n}$ whenever convenient. For $X \subseteq \mathbb{R}^{m+n}$ and $u \in \mathbb{R}^{m}, X_{u}$ denotes the fiber $\left\{x \in \mathbb{R}^{n}:(u, x) \in X\right\}$. (It should be clear from context when subscripts indicate taking fibers, and when they are used as indices.) Given $A \subseteq \mathbb{R}^{l} \times \mathbb{R}^{n}$ and $B \subseteq \mathbb{R}^{m} \times \mathbb{R}^{n}$, put

$$
A \diamond B=\left\{(x, y, z) \in \mathbb{R}^{l+m+n}:(x, z) \in A \&(y, z) \in B\right\} .
$$

(Strictly speaking, we should write something like $\diamond_{l, m, n}$ to reflect the dependence; we will rely upon context instead.) The $\diamond$ operation generalizes both intersection and cartesian products: If $A, B \subseteq \mathbb{R}^{n}$, then, regarding $A, B$ as subsets of $\mathbb{R}^{0} \times \mathbb{R}^{n}$, we have $A \diamond B=A \cap B$; if $A \subseteq \mathbb{R}^{m}$ and $B \subseteq \mathbb{R}^{n}$, then, regarding $A$ as a subset of $\mathbb{R}^{m} \times \mathbb{R}^{0}$ and $B$ as a subset of $\mathbb{R}^{n} \times \mathbb{R}^{0}$, we have $A \diamond B=A \times B$.

Definition. Given $A \subseteq \mathbb{R}^{n}, A \in \mathcal{T}_{n}$ if and only if there exist $m \in \mathbb{N}$, $X \subseteq \mathbb{R}^{m+n}$ definable in $\mathfrak{R}$, and an indexed family $\left(P_{\alpha}\right)_{\alpha \in I}$ of subsets of $E^{m}$ 
such that $A=\bigcup_{\alpha \in I} \bigcap_{u \in P_{\alpha}} X_{u}$.

Every subset of $\mathbb{R}^{n}$ definable in $\mathfrak{R}$ belongs to $\mathcal{T}_{n}$. Moreover, given any $m, P \subseteq E^{m}$ and functions $f_{1}, \ldots, f_{m}: \mathbb{R}^{n} \rightarrow \mathbb{R}$ definable in $\mathfrak{R}$, the set

$$
\left\{x \in \mathbb{R}^{n}:\left(f_{1}(x), \ldots, f_{m}(x)\right) \in P\right\}
$$

belongs to $\mathcal{T}_{n}$, since it is the union of all sets of the form

$$
\left\{x \in \mathbb{R}^{n}: f_{1}(x)=u_{1} \& \ldots \& f_{m}(x)=u_{m}\right\}
$$

where $\left(u_{1}, \ldots, u_{m}\right)$ ranges over $P$.

Claim. $\mathcal{T}_{n}$ is a boolean algebra.

Proof. We show that $\mathcal{T}_{n}$ is closed under complementation and intersection. Let $A \in \mathcal{T}_{n}$. Then there exist $m, X \subseteq \mathbb{R}^{m+n}$, and a family $\left(P_{\alpha}\right)_{\alpha \in I}$ of subsets of $E^{m}$ such that $A=\bigcup_{\alpha \in I} \bigcap_{u \in P_{\alpha}} X_{u}$. Hence,

$$
\mathbb{R}^{n} \backslash A=\bigcap_{\alpha \in I} \bigcup_{u \in P_{\alpha}}\left(\mathbb{R}^{m+n} \backslash X\right)_{u}
$$

and there exists $K \subseteq \prod_{\alpha \in I} P_{\alpha}$ such that

$$
\mathbb{R}^{n} \backslash A=\bigcup_{\gamma \in K} \bigcap_{u \in \gamma(I)}\left(\mathbb{R}^{m+n} \backslash X\right)_{u} .
$$

Let moreover $B \in \mathcal{T}_{n}$. Then there exist $l, Y \subseteq \mathbb{R}^{l+n}$ definable in $\Re$, and a family $\left(Q_{\beta}\right)_{\beta \in J}$ of subsets of $E^{l}$ such that $B=\bigcup_{\beta \in I} \bigcap_{v \in Q_{\beta}} Y_{v}$. Then

$$
A \cap B=\bigcup_{(\alpha, \beta) \in I \times J} \bigcap_{(u, v) \in P_{\alpha} \times Q_{\beta}}(X \diamond Y)_{(u, v)}
$$

(We have established Property (A).)

REMARKS. - In the argument above, note how crucial it is that we allow arbitrary index sets on the unions; there are yet more blatant manifestations of this coming up. • Suppose that $E$ is dense in some interval $I$. Then, for every $n$, every subset of $I^{n}$ belongs to $\mathcal{T}_{n}$. $\bullet$ In general, we do not understand well the relation between the subsets of $\mathbb{R}^{n}$ definable in $(\Re, E)^{\#}$ and the sets in $\mathcal{T}_{n}$. In particular, we do not know if there are sets in $\mathcal{T}_{1}$ that are not definable in $(\Re, E) \#$.

Before defining the collections $\mathcal{S}_{n}$, we give some motivation for the definition. (Here, the reader is assumed to be familiar with the cell decomposition theorem for o-minimal structures; see e.g. [3, Ch. 3] for the statement.) Since $\mathfrak{R}$ is o-minimal, every element of $\mathcal{T}_{n}$ is of the form

$$
\bigcup_{\alpha \in I} \bigcap_{u \in P_{\alpha}}\left(C_{1} \cup \ldots \cup C_{k}\right)_{u}
$$


where $C_{1}, \ldots, C_{k}$ are cells in $\mathbb{R}^{m+n}$ definable in $\mathfrak{R}$, and $m,\left(P_{\alpha}\right)_{\alpha \in I}$ are as usual. We would like to assert that every element of $\mathcal{T}_{n}$ is a finite union of such sets, but with $k=1$. We do not know if this assertion is true; cells are, in general, poorly behaved with respect to boolean operations and permutations of coordinates. So, for present purposes, we loosen the notion of cell.

Definition. A weak cell (with respect to $\mathfrak{R}$ ) in $\mathbb{R}^{n+1}$ is a set of one of the following forms:

(i) $B \times \mathbb{R}$,

(ii) $\left\{(x, t) \in \mathbb{R}^{n+1}: x \in B \& f(x)=t\right\}$,

(iii) $\{(x, t) \in$ $\left.\mathbb{R}^{n+1}: x \in B \& f(x)<t\right\}$, (iv) $\left\{(x, t) \in \mathbb{R}^{n+1}: x \in B \& t<g(x)\right\}$,

(v) $\left\{(x, t) \in \mathbb{R}^{n+1}: x \in B \& f(x)<t<g(x)\right\}$,

where $B \subseteq \mathbb{R}^{n}$ is definable in $\mathfrak{R}$ and $f, g: \mathbb{R}^{n} \rightarrow \mathbb{R}$ are definable in $\mathfrak{R}$.

Clearly, every cell is a weak cell.

Lemma 1. Let $A, B \subseteq \mathbb{R}^{m} \times \mathbb{R}^{n+1}$ be weak cells. Then $A \diamond B$ is a weak cell in $\mathbb{R}^{2 m+n+1}$.

We leave the proof of the above to the reader. (Just check all the possible combinations.)

Definition. For $A \subseteq \mathbb{R}^{n+1}, A \in \mathcal{S}_{n+1}$ if and only if there exist $m \in \mathbb{N}$, a weak cell $C \subseteq \mathbb{R}^{m+n+1}$ and an indexed family $\left(P_{\alpha}\right)_{\alpha \in I}$ of subsets of $E^{m}$ such that $A=\bigcup_{\alpha \in I} \bigcap_{u \in P_{\alpha}} C_{u}$. (Put $\mathcal{S}_{0}:=\left\{\mathbb{R}^{0}, \emptyset\right\}$.) We will refer to the sets in $\mathcal{S}_{n+1}$ arising from each of the five forms of weak cells as being of types (i) through (v) respectively.

Next, we state an elementary (but somewhat tedious) technical result, the verification of which we leave to the reader.

Lemma 2. Let $C_{1}, \ldots, C_{k+1} \subseteq \mathbb{R}^{m} \times \mathbb{R}^{n}$ and $\left(P_{\alpha}\right)_{\alpha \in I}$ be a family of subsets of $\mathbb{R}^{m}$. Then $\bigcup_{\alpha \in I} \bigcap_{u \in P_{\alpha}}\left(C_{1} \cup \ldots \cup C_{k+1}\right)_{u}$ is equal to the union of the three sets

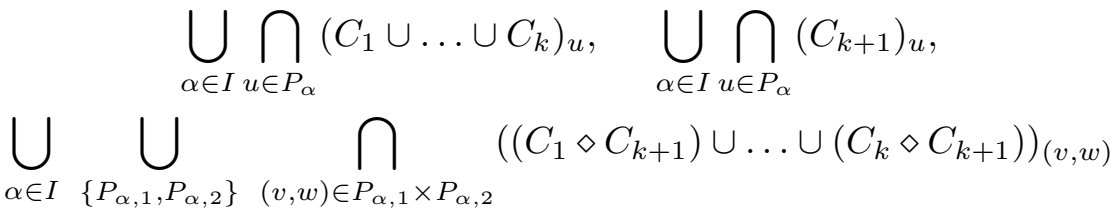

where, for $\alpha \in I,\left\{P_{\alpha, 1}, P_{\alpha, 2}\right\}$ ranges over all 2-element partitions of $P_{\alpha}$.

We are now ready to establish Property (В).

Claim. Every element of $\mathcal{T}_{n}$ is a finite union of elements of $\mathcal{S}_{n}$.

Proof. Use Lemmas 1 and 2 to show the following by induction on $k \geq 1$ : If $m \in \mathbb{N}, C_{1}, \ldots, C_{k} \subseteq \mathbb{R}^{m+n+1}$ are weak cells in $\mathbb{R}^{m+n+1}$, and $\left(P_{\alpha}\right)_{\alpha \in I}$ is a family of subsets of $E^{m}$, then $\bigcup_{\alpha \in I} \bigcap_{u \in P_{\alpha}}\left(C_{1} \cup \ldots \cup C_{k}\right)_{u}$ is a finite union of elements of $\mathcal{S}_{n+1}$. The result then follows from cell decomposition (in $\mathfrak{R}$ ). 
REMARK. We have not yet used that we are working over the ordered additive group of real numbers.

Next, we record two easy, but crucial, observations about sets of real numbers. We omit the proofs.

Lemma 3. Let $\emptyset \neq S \subseteq \mathbb{R}$. Then:

- $\sup S<\infty$ if and only if there exists $s \in S$ such that $s^{\prime}<s+1$ for all $s^{\prime} \in S$. $\bullet$ inf $S>-\infty$ if and only if there exists $s \in S$ such that $s<s^{\prime}+1$ for all $s^{\prime} \in S . \bullet \inf S>0$ if and only if there exists $s \in S$ such that $0<s<2 s^{\prime}$ for all $s^{\prime} \in S$.

Lemma 4. Let $\emptyset \neq A, B \subseteq \mathbb{R}$. The following are equivalent:

- There exists $t \in \mathbb{R}$ such that $a<t<b$ for all $a \in A$ and $b \in B$. $a<b$ for all $a \in A$ and $b \in B$; moreover, $\max A$ does not exist, or $\min B$ does not exist, or there exist $a \in A$ and $b \in B$ such that $b-a<2\left(b^{\prime}-a^{\prime}\right)$ for all $a^{\prime} \in A$ and $b^{\prime} \in B$.

We are now ready to establish Property (c).

Claim. The projection on the first $n$ variables of an element of $\mathcal{S}_{n+1}$ belongs to $\mathcal{T}_{n}$.

Proof. Let $A=\bigcup_{\alpha \in I} \bigcap_{u \in P_{\alpha}} C_{u}$ where $C \subseteq \mathbb{R}^{m+n+1}$ is a weak cell and $\left(P_{\alpha}\right)_{\alpha \in I}$ is a family of subsets of $E^{m}$. We must exhibit $A^{\prime} \in \mathcal{T}_{n}$ such that for all $x \in \mathbb{R}^{n}$ we have $x \in A^{\prime}$ if and only if there exists $t \in \mathbb{R}$ with $(x, t) \in A$. We adopt the following notation: For any set $Y$, we write $\bigvee_{y \in Y}$ instead of $\exists y \in Y$, and $\bigwedge_{y \in Y}$ instead of $\forall y \in Y$. If $A$ is of type (i), then $C=B \times \mathbb{R}$ for some $B \subseteq \mathbb{R}^{m+n}$ definable in $\mathfrak{R}$. Then $A=\left(\bigcup_{\alpha \in I} \bigcap_{u \in P_{\alpha}} B_{u}\right) \times \mathbb{R}$, and the result is clear. We sketch the rest of the proof, leaving routine details to the reader. Fix $x \in \mathbb{R}^{n}$.

Suppose $A$ is of type (ii). Then

$$
\begin{aligned}
\bigvee_{t \in \mathbb{R}} \bigvee_{\alpha \in I} \bigwedge_{u \in P_{\alpha}}[(u, x) & \in B \& f(u, x)=t] \\
& \Leftrightarrow \bigvee_{\alpha \in I} \bigvee_{v \in P_{\alpha}} \bigwedge_{u \in P_{\alpha}}[(u, x) \in B \& f(u, x)=f(v, x)] .
\end{aligned}
$$

Suppose $A$ is of type (iii). Let $\alpha \in I$. By Lemma 3,

$$
\sup f\left(P_{\alpha} \times\{x\}\right)<\infty \Leftrightarrow \bigvee_{v \in P_{\alpha}} \bigwedge_{u \in P_{\alpha}}[f(u, x)<f(v, x)+1]
$$

Hence

$$
\bigvee_{t \in \mathbb{R}} \bigvee_{\alpha \in I} \bigwedge_{u \in P_{\alpha}}[(u, x) \in B \& f(u, x)<t]
$$




$$
\Leftrightarrow \bigvee_{\alpha \in I} \bigvee_{v \in P_{\alpha}} \bigwedge_{u \in P_{\alpha}}[(u, x) \in B \& f(u, x)<f(v, x)+1] .
$$

(The type (iv) case is similar.) Suppose that $A$ is of type (v). Let $\alpha \in I$. By Lemma 4, we have

$$
\bigvee_{t \in \mathbb{R}} \bigwedge_{u \in P_{\alpha}}[f(u, x)<t<g(u, x)]
$$

if and only if

$$
\bigwedge_{u, v \in P_{\alpha}} f(u, x)<g(v, x)
$$

and at least one of the following holds:

$$
\begin{aligned}
& \bigwedge_{u \in P_{\alpha}} \bigvee_{v \in P_{\alpha}}[f(u, x)<f(v, x)], \\
& \bigwedge_{u \in P_{\alpha}} \bigvee_{v \in P_{\alpha}}[g(v, x)<g(u, x)], \\
& \bigvee_{u, v \in P_{\alpha}} \bigwedge_{u^{\prime}, v^{\prime} \in P_{\alpha}}\left[g(v, x)-f(u, x)<2\left(g\left(v^{\prime}, x\right)-f\left(u^{\prime}, x\right)\right)\right] .
\end{aligned}
$$

Finish as in the earlier cases (using also the fact that $\mathcal{T}_{n}$ is a boolean algebra).

We establish a stronger version of Property (D) that we hope will be helpful in future investigations.

Claim. Let $A \in \mathcal{S}_{n+1}$. Suppose that, for every $x \in \mathbb{R}^{n}, A_{x}$ has no interior. Then there exist $m$ and $f: \mathbb{R}^{m+n} \rightarrow \mathbb{R}$, definable in $\mathfrak{R}$, such that for every $x \in \mathbb{R}^{n}$ we have $A_{x} \subseteq \operatorname{cl}\left(f\left(E^{m} \times\{x\}\right)\right)$.

(Property (D) is just the case $n=0$.)

Proof. Let $\emptyset \neq A \in \mathcal{S}_{n+1}$ be such that $A_{x}$ has no interior for every $x \in \mathbb{R}^{n}$; then $A$ must be of type (ii) or of type (v). Suppose $A$ is of type (ii). Then there exist $m$ and $f: \mathbb{R}^{m+n} \rightarrow \mathbb{R}$ definable in $\mathfrak{R}$ such that for each $x \in \mathbb{R}^{n}$, either $A_{x}=\emptyset$ or $A_{x}=f(P \times\{x\})$ for some $P$ (depending on $x$ ) contained in $E^{m}$. Suppose $A$ is of type (v). Then there exist $m, B \subseteq \mathbb{R}^{m+n}$, and functions $f, g: \mathbb{R}^{m+n} \rightarrow \mathbb{R}$ definable in $\mathbb{R}$ such that for all $x \in \mathbb{R}^{n}$, we have

$$
A_{x}=\bigcup_{\alpha \in I} \bigcap_{u \in P_{\alpha}}\{t \in \mathbb{R}:(u, x) \in B \& f(u, x)<t<g(u, x)\} .
$$

Let $x \in \mathbb{R}^{n}$. Since $A_{x}$ has no interior, each set

$$
\bigcap_{u \in P_{\alpha}}\{t \in \mathbb{R}:(u, x) \in B \& f(u, x)<t<g(u, x)\}
$$


has no interior. Hence,

$$
A_{x} \subseteq\left\{\sup f\left(P_{\alpha} \times\{x\}\right): \alpha \in I\right\} \cap \mathbb{R} \subseteq \operatorname{cl}\left(f\left(E^{m}\right) \times\{x\}\right) .
$$

(This ends the proof of Theorem A.)

Some remarks on sparseness. We noted previously that every subset of $\mathbb{R}$ having cardinality less than that of $\mathbb{R}$ is sparse with respect to every expansion of the real line. We discuss here some other examples (and counterexamples).

1. There are subsets of $\mathbb{R}$ having the cardinality of the continuum that are sparse with respect to every o-minimal expansion of $(\mathbb{R},<,+)$. First, let $E \subseteq \mathbb{R}$ be such that, for every positive integer $n, E^{n}$ has Hausdorff dimension 0 . We show that $E$ is sparse with respect to every o-minimal expansion of $(\mathbb{R},<,+)$. To see this, let $f: \mathbb{R}^{n} \rightarrow \mathbb{R}$ be such that $(\mathbb{R},<,+, f)$ is o-minimal. By Peterzil et al. $[5],(\mathbb{R},+, \cdot, f)$ is o-minimal. By $C^{1}$ cell decomposition [3, Ch. 7], there is a finite partition $\mathcal{C}$ of $\mathbb{R}^{n}$ into locally closed sets such that each restriction $f \mid C: C \rightarrow \mathbb{R}(C \in \mathcal{C})$ is $C^{1}$. Each $E^{n} \cap C$ has Hausdorff dimension 0 , and $f \mid C$ is Lipschitz on compact subsets of $C$, so each $f\left(E^{n} \cap C\right)$ has Hausdorff dimension 0. Hence, $f\left(E^{n}\right)$ has Hausdorff dimension 0 (and thus has no interior). Now we need only exhibit $E \subseteq \mathbb{R}$ such that $E$ has the cardinality of the continuum and each power $E^{n}$ has Hausdorff dimension 0 . Such sets are easily obtained by using infinite series methods; for example, let $E_{0}$ be the set of all real numbers of the form $\sum_{m=1}^{\infty} a_{m} 2^{-m !}$ with each $a_{m} \in\{0,1\}$ (cf. Oxtoby's treatment of Liouville numbers [4, Chapter 2]).

Remark. We do not know if $\left(\mathbb{R},+, \cdot, E_{0}\right)$ satisfies the "interior or nowhere dense" condition on its definable sets.

2. In contrast to the above, there are compact, nowhere dense, null subsets of $\mathbb{R}$ that are not sparse with respect to any expansion of $(\mathbb{R},<,+)$ : the classic middle-thirds Cantor set is an example (its difference set has interior).

3. If $E$ is $\mathfrak{R}$-sparse and has the Baire property, then $E$ is meager (otherwise, the difference set has interior; see e.g. [4, Thm. 4.8]). Similarly, if $E$ is measurable and $\mathfrak{R}$-sparse, then $E$ is null.

4. It is easy to see that every proper $\mathbb{Q}$-linear subspace of $\mathbb{R}$, as well as every $\mathbb{Q}$-linear basis of $\mathbb{R}$, is sparse with respect to $(\mathbb{R},<,+)$. (Hence, in contrast to items 1 and 3 above, there are "large" sparse sets.) More generally, if $E$ is the underlying set of a proper elementary substructure of $\mathfrak{R}$, then $E$ is $\mathfrak{R}$-sparse; see [2, Lemma 4.1]. 
5. We think that the assumption of sparseness is necessary in Theorem $\mathrm{B}$, that is, we conjecture that there exist $A \subseteq B \subseteq \mathbb{R}$ such that $B$ is nowhere dense, every subset of $\mathbb{R}$ definable in $(\mathbb{R},+, \cdot, B)$ has interior or is nowhere dense, and $(\mathbb{R},+, \cdot, A)$ defines dense, codense subsets of $\mathbb{R}$. (Candidates for $B$ include suitably randomized fat Cantor sets.)

\section{References}

[1] L. van den Dries, The field of reals with a predicate for the powers of two, Manuscripta Math. 54 (1985), 187-195.

[2] —, Dense pairs of o-minimal structures, Fund. Math. 157 (1998), 61-78.

[3] - Tame Topology and o-Minimal Structures, London Math. Soc. Lecture Note Ser. 248, Cambridge Univ. Press, Cambridge, 1998.

[4] J. Oxtoby, Measure and Category, Grad. Texts in Math. 2, Springer, New York, 2nd ed., 1980.

[5] Y. Peterzil, P. Speissegger and S. Starchenko, Adding multiplication to an o-minimal expansion of the additive group of real numbers, in: Logic Colloquium '98, Lecture Notes in Logic 13, A. K. Peters, Natick, MA, 2000, 357-362.

Department of Mathematics

The Ohio State University

231 West 18th Avenue

Columbus, OH 43210, U.S.A.

E-mail: friedman@math.ohio-state.edu

miller@math.ohio-state.edu

Received 7 September 1999;

in revised form 22 August 2000 